\title{
Ameliorating Effects of Sphingomyelin-Based Liposomes on Sarcopenia in Senescence-Accelerated Mice
}

\author{
Yuuki Ishida, Yuri Kiyokawa, Tomohiro Asai, and Naoto Oku* \\ Department of Medical Biochemistry, Graduate School of Pharmaceutical Sciences, University of Shizuoka; 52-1 \\ Yada, Suruga-ku, Shizuoka 422-8526, Japan. \\ Received November 17, 2015; accepted January 16, 2016
}

The effects of orally administered sphingomyelin-based liposomes (SM-lipo) on muscle function were investigated in senescence-accelerated mice prone 1 (SAMP1) for the purpose of protection against or treatment of sarcopenia. SM-lipo were prepared by thin lipid-film hydration followed by extrusion. Their spherical shape was observed by transmission electron microscopy. The obtained liposomes were stable in gastric liquid and intestinal fluid models as well as in water. In in vitro tests liposomalization of sphingomyelin significantly increased its transport into human intestinal epithelial Caco-2 cells. In addition, SM-lipo upregulated the proliferation of murine $\mathrm{C} 2 \mathrm{C} 12$ myoblasts compared with free sphingomyelin or phosphatidylcholine-based liposomes (PC-lipo). Finally, SM-lipo orally administered to SAMP1 for 10 weeks significantly increased quadriceps femoris weight and extended swimming time until fatigue compared with PC-lipo. In conclusion, these findings indicate that SM-lipo are well absorbed into the body and improve muscle weakness caused by senescence.

Key words sarcopenia; sphingomyelin; senescence-accelerated mouse; liposome; muscle

Loss of muscle mass induces a progressive decline in physical performance; and it also leads to decrease in quality of life, including increased risk of disability and mortality. ${ }^{1)}$ This age-related deficit is known as sarcopenia. Sarcopenia is a progressive, insidious process characterized by a $3-8 \%$ reduction in lean muscle mass. The etiology of sarcopenia is multi-factorial and involves both intrinsic and extrinsic factors. $^{2,3)}$ Increasing muscle mass is important to prevent sarcopenia. The promising approaches for increasing muscle mass are exercise training and proper nutrition. Exercise training is generally accepted as a useful strategy to increase the number of mitochondria and the expression of mitochondria-associated proteins, as well as to improve the function of mitochondria in muscle. ${ }^{4)}$ Food nutrients such as sphingolipids, leucine, creatine, and whey protein are known to be involved in increasing muscle mass. ${ }^{5-7)}$

Sphingolipids were first characterized by Thudichum, who studied the chemical constituents of brain tissue; whereupon he named their novel and characteristic "sphingosine" backbone for "the many enigmas it has presented to the inquirer."8) Most foods contain sphingolipids. Humans on an ordinary Western diet ingest $0.3-0.4 \mathrm{~g}$ of sphingolipids per day, of which sphingomyelin in meat, milk, egg products, and soybeans is a large part. $^{9-11)}$ Also, sphingolipids are highly bioactive molecules present mainly in polar lipids of animal origin $^{9)}$ and the second most abundant polar lipid next to phosphatidylcholine in plasma lipoproteins ${ }^{12)}$; and they are associated with cellular signaling, insulin resistance, metabolic disease, dementia, and so on. ${ }^{13,14)}$ As a type of sphingolipid, sphingomyelin in mammalian cells is co-localized with cholesterol mainly in the plasma membrane and in lysosomal and Golgi membranes. Dietary supplementation with sphingomyelin combined with exercise increases the swimming time of mice and the level of peroxisome proliferator-activated receptor gamma coactivator $1 \alpha$ (PGC- $1 \alpha)$ mRNA expression in the soleus muscle of mice. $\left.{ }^{15}\right)$ It was shown in mice that sphingosine-1-phosphate, a product of sphingomyelin metabolism, promotes skeletal muscle regeneration. ${ }^{16,17)}$ In addition, sphingosine-1-phosphate stimulates the growth of human airway smooth muscle cells and increases in muscle contractile force in mice. ${ }^{18,19)}$ These findings suggest that one of mechanisms underlying the ameliorating effects of sphingomyelin on sarcopenia can be explained by signaling of sphingosine1-phosphate.

Although dietary sphingomyelin is not absorbed intact, it is metabolized to ceramide, sphingosine, and fatty acids in the gut. ${ }^{20)}$ Sphingomyelin digestion is an extended process with limited capacity and is catalyzed primarily by alkaline sphingomyelinase and neutral ceramidase, which are in part released into and act in the gut lumen and, therefore, sphingomyelin absorption is limited. ${ }^{20,21)}$ Studies on lymphatic duct-cannulated rats that were fed $\left[{ }^{14} \mathrm{C}\right]$ stearic acid-labeled sphingomyelin showed that little radio-labeled sphingomyelin was absorbed intact to the chyle. ${ }^{22)}$

Liposomes, which are lipid bilayer vesicles composed of amphipathic phospholipids, have been used as delivery systems for a wide variety of biologically active substances. We previously reported that oral delivery of insulin into the bloodstream was achieved by its liposomalization. ${ }^{23)}$ Liposomes have been found to be useful for effective absorption in the small intestines. ${ }^{24,25)}$ Also, the preparation of sphingomyelin-based liposomes (SM-lipo) was achieved earlier ${ }^{26)}$ and was applied to the basal layer or stratum corneum side of a 3D; three-dimensional culture human skin model. ${ }^{27)}$ Furthermore, liposomes with phosphatidylethanol were also reported as a carrier for oral delivery of insulin. ${ }^{28)}$ The physicochemical properties of sphingomyelin are similar to those of saturated phosphatidylcholine. ${ }^{26)}$ Therefore, it is possible to use sphingomyelin as a component for liposome dispersions, which might be advantageous for both oral delivery and effective absorption.

In the present study, we sought to enhance the absorption of 
sphingomyelin and boost its effectiveness by liposomalization in senescence-accelerated mice prone 1 (SAMP1) mice. The findings from this study will help in the application of SMlipo in the fields of foods and drugs.

\section{MATERIALS AND METHODS}

Preparation of Liposomes Sphingomyelin from milk and dipalmitoylphosphatidylcholine (DPPC) were purchased from the NOF Corporation (Tokyo, Japan). Cholesterol was purchased from Sigma-Aldrich (St. Louis, MO, U.S.A.). All other chemicals and solvents were analytical grade. SM-lipo and phosphatidylcholine-based liposomes (PC-lipo) were prepared by thin lipid-film hydration followed by extrusion. In brief, sphingomyelin and cholesterol (7:3 as a molar ratio) dissolved in chloroform were transferred to a round-bottomed flask, evaporated until a thin lipid film had formed under reduced pressure, and stored in vacuo for at least $1 \mathrm{~h}$. The dried film was hydrated with water, warmed at $65^{\circ} \mathrm{C}$ in a water bath, vortexed until the lipids had become detached from the side of the flask, and sonicated with a bath-type sonicator at $65^{\circ} \mathrm{C}$. Three cycles of the following were performed: freezing of the liposomal solution in the flask with liquid nitrogen, thawing at room temperature, incubating at $65^{\circ} \mathrm{C}$ in a water bath for $5 \mathrm{~min}$, and vortexing for $30 \mathrm{~s}$. Then, the liposomes were extruded through polycarbonate membrane filters having 100$\mathrm{nm}$ pores by use of an Extruder (Lipex, Vancouver, Canada) kept at $65^{\circ} \mathrm{C}$.

Characterization of Liposomes The particle diameter, $\zeta$-potential, and polydispersity index (PDI) of the liposomes were determined with a Zeta Sizer (Nano-ZS; Malvern Instruments, Worcs, U.K.) after they had been diluted in phosphate-buffered saline, $\mathrm{pH} 7.4$ (PBS). The structure of these liposomes was analyzed by transmission electron microscopy (TEM, JEM 2000; Jeol, Tokyo, Japan) after having been negatively stained with uranyl acetate solution.

Studies on Diffusion/Permeation of Sphingomyelin in Caco-2 Cells The Caco-2 cell line was derived from a heterogeneous human epithelial colorectal adenocarcinoma. Although this cell line originated from a carcinoma, the cells become differentiated and polarized by being cultured under specific conditions, resulting in cells that resemble the enterocytes lining the small intestine. ${ }^{29,30)}$ Caco-2 cell monolayers were prepared by cultivating Caco- 2 cells for 3 weeks on polycarbonate membrane filters equipped on camber insert. The pore size of the membrane filters was $0.4 \mu \mathrm{m}$; and the diameter, $24 \mathrm{~mm}$. The electrical resistances of the Caco- 2 cell lines were measured over $300 \Omega \mathrm{cm}$ at room temperature in Dulbecco's modified Eagle's medium (DMEM). The integrity of the membrane was also checked after each experiment by measuring the electrical resistance. The Caco-2 cell monolayer on the membrane filter was placed between the donor (upper layer) and receptor (lower layer) compartments of a 24-well culture plate and used as a model of human intestinal absorption of drugs and other compounds. Sphingomyelin suspended in DMEM or SM-lipo in the medium was added to the upper layer (total sphingomyelin concentration of $3 \mathrm{~mm}$ ), and the receptor compartment was filled with sphingomyelin-free medium. The samples were collected from the donor compartment, receptor one, and cell fraction after a 90-min incubation. Then the amounts of sphingomyelin in these samples were deter- mined by HPLC (Chromaster, Hitachi, Japan). In the case of the cell fraction, SM was solubilized with Triton X-100 before being applied to HPLC. All experiments were carried out in triplicate.

Quantification of Sphingomyelin The sphingolipid extracts were dissolved in methanol, mixed, and sonicated at room temperature for $10 \mathrm{~min}$. The HPLC analysis was performed with a phase column containing high-purity silica gel (Inertsil SIL-100A; GL Science, Japan). The isocratic mobile phase was composed of acetonitrile-methanol-distilled water $(65: 21: 14)$ with $0.1 \%(\mathrm{v} / \mathrm{v})$ phosphoric acid, and the flow rate $(1.3 \mathrm{~mL} / \mathrm{min})$ was accurately regulated. The UV detector was set at $210 \mathrm{~nm}^{31)}$

Cell Culture and Proliferation Assay Murine $\mathrm{C} 2 \mathrm{C} 12$ myoblasts (EC91031101-F0) were purchased from the European Collection of Cell Cultures (Dainippon Sumitomo Pharma Biomedical, Japan). The cells were plated onto flexible-bottomed plates previously coated with $1 \mathrm{mg} / \mathrm{mL}$ poly-Llysine (Sigma-Aldrich) and fibronectin (Sigma-Aldrich), and maintained in $5 \% \mathrm{CO}_{2}$ at $37^{\circ} \mathrm{C}$ in DMEM supplemented with $10 \%$ fetal bovine serum (Sigma-Aldrich). Murine C2C12 cells were seeded ( 1000 cells/well) into each well of a 96-well plate and incubated overnight in a $\mathrm{CO}_{2}$ incubator. After a change of the medium, the cells were incubated at $37^{\circ} \mathrm{C}$ for $72 \mathrm{~h}$ in the presence of $125 \mu \mathrm{M}$ sphingomyelin dispersed in medium, SMlipo, or PC-lipo. Then the viability of the cells was measured by performing the WST assay (Roche, Basel, Switzerland) according to the manufacturer's instruction.

Animals and Experimental Design Seven-week-old male SAMP1 were purchased from Japan SLC, Inc. (Hamamatsu, Japan) and maintained under controlled temperature, humidity, and lighting. The mice had free access to feeding and drinking water for a week. At the age of 9 weeks, all mice were weighed and measured swimming time until fatigue. Then they were divided into 3 groups, namely, the control group, which drank water; the SM group, which drank $2 \mathrm{~mm}$ SM-lipo; and the PC group, which drank $2 \mathrm{~mm}$ PC-lipo ( $n=6$ per group, 3 mice per cage). From the age of 10 weeks, the mice were given SM-lipo, PC-lipo or normal water for 10 weeks (until 20 weeks old). These test solutions were exchanged for fresh ones every week. The volumes of liposome solutions and water, as well as the weight of the mice, were monitored every 2 weeks; and the swimming time until fatigue was measured every 5 weeks. None of the mice performed any specified exercise. Animal care and experiments were performed in accordance with the Guidelines for the Care and Use of Laboratory Animals of the University of Shizuoka. All animal procedures were approved by the Animal and Ethics Review Committee of the University of Shizuoka.

Biochemical Analysis Plasma was obtained from the blood of the mice by centrifugation at $3500 \times \mathbf{g}$ for $15 \mathrm{~min}$. Plasma glucose, triglycerides (TG), and non-esterified fatty acid (NEFAs) were quantified by using Autokit Glucose (Wako Chemicals, USA, Inc., Richmond, VA, U.S.A.), TG E Test Wako (Wako Pure Chemical Industries, Ltd., Osaka, Japan) and NEFA-C Test Wako (Wako Pure Chemical Industries, Ltd.), respectively. All measurements were performed in accordance with the manufacturer's instructions.

Statistical Analysis All values were presented as the mean \pm standard deviation (S.D.). Unpaired Student's $t$-tests after $F$-test of the homogeneity of within-group variance were 
used to compare values between 2 groups. When more than 2 groups were compared, statistical analysis was conducted with one-way ANOVA followed by Fisher's protected least significant difference or Dunnett's post-hoc tests (Statview for Windows version 5.0; SAS Institute Inc., Cary, NC, U.S.A.). $p<0.05$ was considered as being statistically significant.

\section{RESULTS}

Stability of SM-lipo The particle diameter, zeta potential, and PDI of SM-lipo were $132.9 \mathrm{~nm},-3.0 \mathrm{mV}$, and 0.139 , respectively. Their spherical shape structure was observed by negative-staining TEM (Fig. 1). The liposomes were quite stable at room temperature at least for $30 \mathrm{~d}$. Stability of SMlipo in water, PBS, gastric liquid, and intestinal fluid models was examined. The particle diameter and zeta potential of the liposomes were little changed for at least $48 \mathrm{~h}$ (Fig. 2). These data suggest that SM-lipo was stable in gastrointestinal tract (GI tract) after oral administration.

SM-lipo Penetration through Caco-2 Cell Layer The percent penetration of sphingomyelin through Caco-2 cells $(n=3)$ by use of SM-lipo was $15.4 \%$; whereas sphingomyelin, which dispersed in the medium, didn't penetrate at all after incubation at $37^{\circ} \mathrm{C}$ for $90 \mathrm{~min}$. Additionally, $3.5 \%$ of the SM-lipo was taken up by the Caco-2 cells, although sphingomyelin was little absorbed (Fig. 3). Simultaneously, the donor chamber volume for SM-lipo was $85.6 \%$; and that for sphingomyelin, $96.5 \%$.

We also demonstrated the penetration of sphingomyelin by using the Parallel Artificial Membrane Permeability Assay (PAMPA). PAMPA is performed in a 96-well format, and rapid quantification can be achieved by using a UV plate reader. In the PAMPA study, the percent membrane absorption of SM-lipo was $41.8 \%$ for $24 \mathrm{~h}$, whereas that of sphingomyelin was $9.4 \%$ (Supplementary Fig. 1).

A

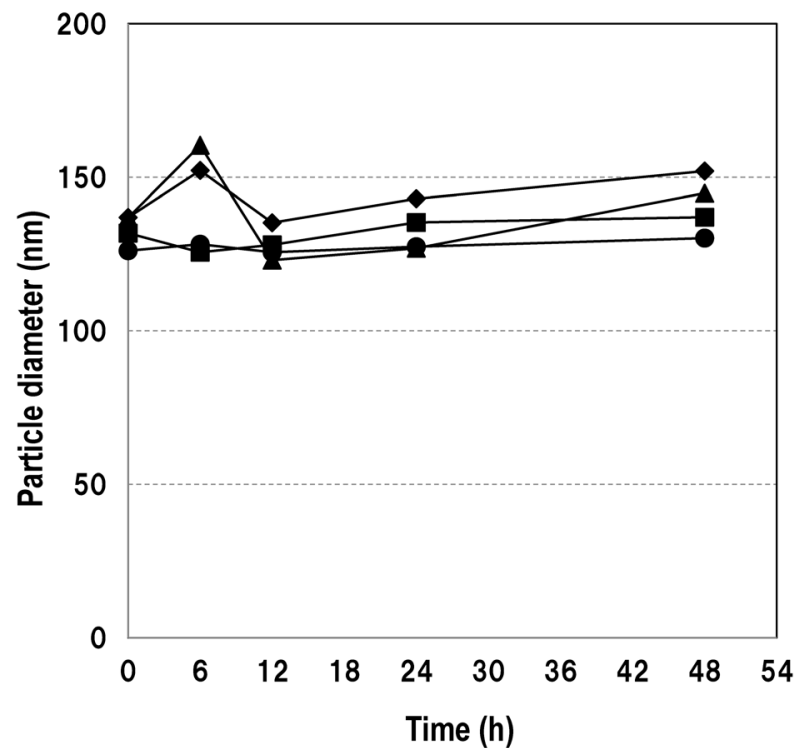

Effect of SM-lipo on the Proliferation of Murine C2C12 Myoblasts Treatment of murine $\mathrm{C} 2 \mathrm{C} 12$ myoblasts with SMlipo significantly enhanced cell proliferation compared with that for all other groups (Fig. 4). The cell number was increased approximately 1.2-fold by SM-lipo treatment for $72 \mathrm{~h}$. In contrast, no significant difference in the number of proliferating cells was observed among sphingomyelin, PC-lipo, and water treatment groups.

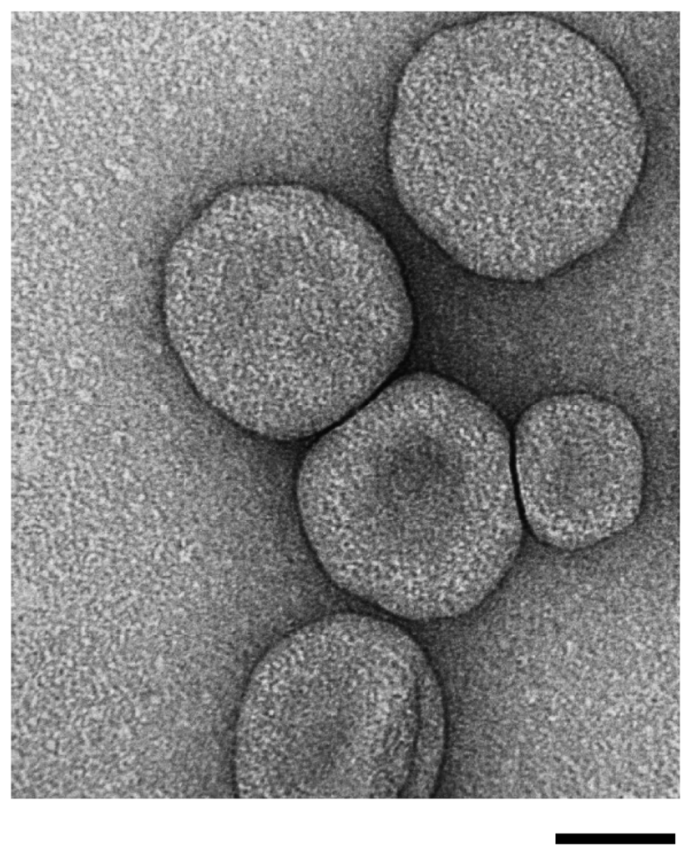

Fig. 1. Negative-Staining TEM Image of Sphingomyelin:Cholesterol (7:3 as Molar Ratio)-Based Liposomes

Scale bar, $100 \mathrm{~nm}$.

\section{B}

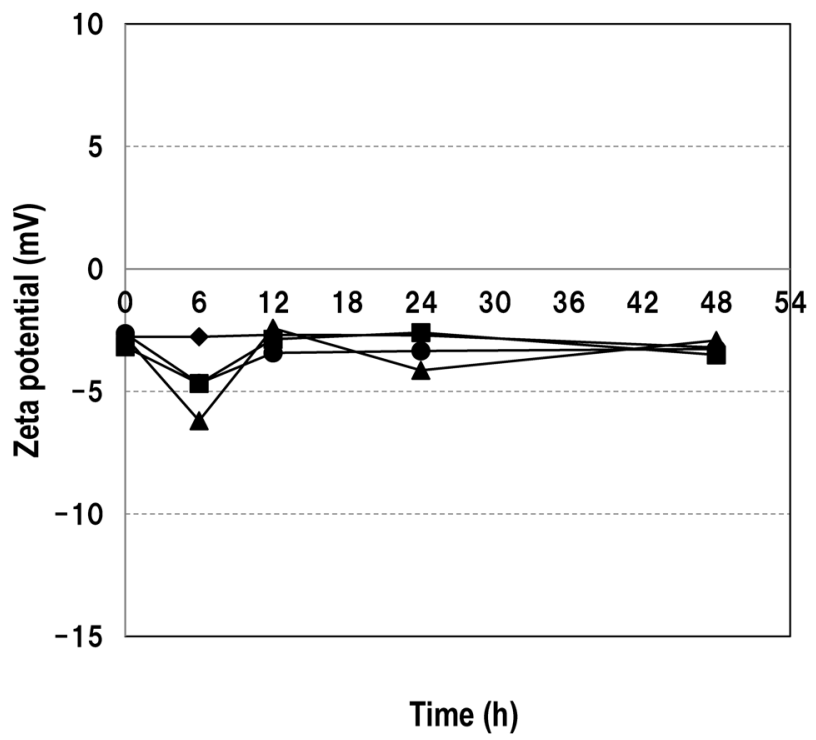

Fig. 2. Stability of Sphingomyelin-Based Liposomes

SM-lipo were incubated in water $(\bullet)$, PBS $(\mathbf{\square})$, gastric liquid model $(\boldsymbol{\Delta})$ or intestinal fluids model $(\bullet)$ at $25^{\circ} \mathrm{C}$. Changes in the particle diameter (a) and zeta potential $(\mathrm{b})$ of the liposomes during incubation were monitored. 


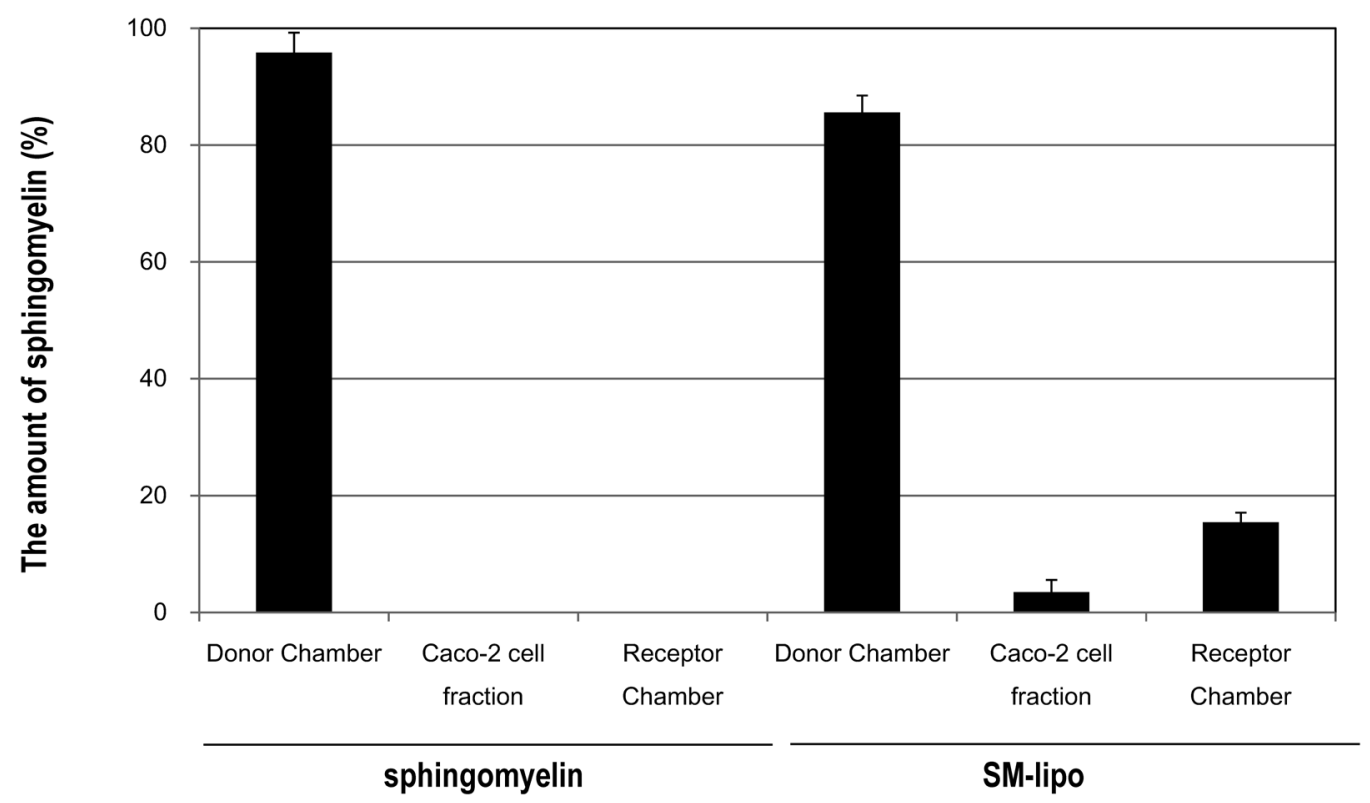

Fig. 3. Penetration or Absorption of Sphingomyelin or Sphingomyelin-Based Liposome into Caco-2 Cells

Sphingomyelin dispersed in DMEM or SM-lipo in DMEM were added to the donor compartment, where Caco-2 cells were cultured on a membrane filter of the filterequipped insert; and the receptor compartment was filled with sphingomyelin-free DMEM. The samples were collected from the donor and receptor compartments and cell fraction after 90-min incubation. Then, the amount of sphingomyelin in each sample was determined with HPLC. The experiment was repeated 3 times, and results are shown as the mean \pm S.D. of these experiments taken together.

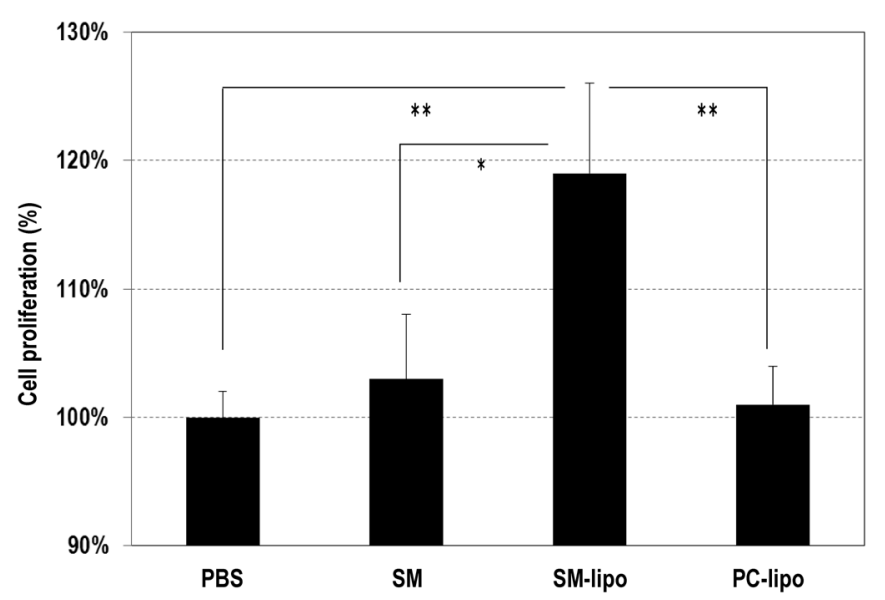

Fig. 4. Effect of Sphingomyelin or Sphingomyelin-Based Liposomes on the Proliferation of Myoblast Cells

$\mathrm{C} 2 \mathrm{C} 12$ myoblasts were incubated in the presence of sphingomyelin, SM-lipo or PC-lipo for $72 \mathrm{~h}$. The same volume of PBS as that of the other samples was added to the serum-containing DMEM as a control. Then the number of viable cells was determined by performing the WST assay. The experiment was repeated 3 times, and results are shown as the mean \pm S.D. of these experiments taken together. Significant difference are shown as $* * p<0.05$ and $* * p<0.01$

Effect of SM-lipo on Muscle in SAMP1 SAMP1 were given water containing SM-lipo, PC-lipo or water alone ad libitum for 10 weeks. During this period, there was no difference in water or food intake or in body weight change among the groups treated without or with water containing liposomes (Supplementary Fig. 2). The SM-lipo-treated SAMP1 tended to show extended swimming time until fatigue compared with the PC-lipo- or plain water-treated groups (Fig. 5). These data showed a certain trend toward significance $(p=0.08)$. Additionally, SAMP1 that had ingested SM-lipo had a significantly increased weight of their quadriceps femoris muscle compared with PC-lipo- or plain water-treated groups (Fig. 6). The weight of the quadriceps femoris muscle increased approximately 1.5-fold in SM-lipo-treated group compared with that for the control. On the other hand, the weight of soleus muscle and gastrocnemius muscle showed no significant increase (Figs. 6A, B). Interestingly, SAMP1 that had ingested PC-lipo showed a significant increase in the weight of their quadriceps femoris muscle compared with the given plain water (Fig. 6C). Blood component levels (glucose, TG, and NEFAs) were hardly changed after liposome intake (data not shown).

\section{DISCUSSION}

Sarcopenia is a disease associated with loss of muscle strength and function during the aging process. Because of increasing aging of the population in recent years, especially in developed countries, medical bills related with sarcopenia have become problematic throughout the world, especially in those countries. Sarcopenia is thought to affect $30 \%$ of individuals over 60 years of age and more than $50 \%$ of those over 80 years. ${ }^{32)}$ When the elderly suffer from both sarcopenia and osteoporosis, the risk of falling and subsequent fracture incidence is increased. ${ }^{33)}$ Therefore, appropriate treatment of sarcopenia should help to lessen its burden on co-existing disease. Although the biochemical and molecular mechanisms responsible for the loss of muscle during aging are not fully understood, ${ }^{2,3}$ the loss of muscle during aging is known to be the result of both decreased protein synthesis and increased protein degradation. ${ }^{34,35)}$

Currently, protection against or prevention of sarcopenia is exercise and eating proper nutrition. Exercise, specifically resistance training or strength training, increases muscle strength and endurance. Resistance training has been reported to positively influence the neuromuscular system, hormone concentration, and rate of protein synthesis. ${ }^{36,37)}$ Eating a proper nutrition is also important to build muscle. Dietary proteins are recommended to prevent sarcopenia. To maximize 


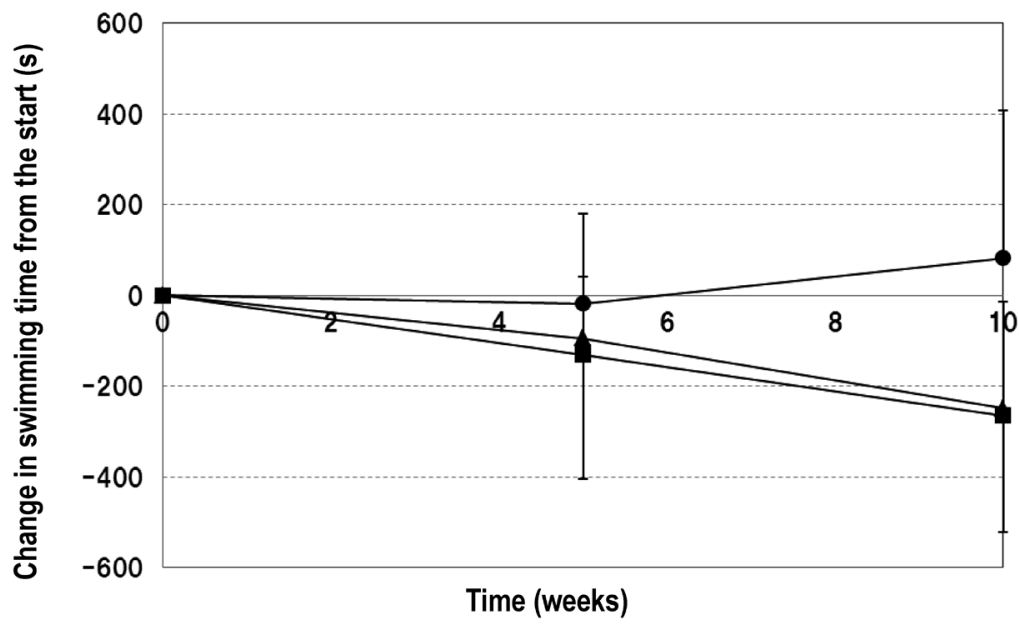

Fig. 5. Effect of Sphingomyelin-Based Liposomes on Swimming Endurance

SAMP1 mice (10 weeks old) were reared for an additional 5 or 10 weeks with standard chow and plain drinking water (ם) or drinking water containing 2 mM SM-lipo ) or 2 mм PC-lipo $(\boldsymbol{\Delta})$. Swimming time of the mice $(n=6)$ was determined before ( 0 week) or after 5 and 10 weeks of intake. Swimming-time changes are shown as the mean \pm S.D., where the control swimming time was about $700 \mathrm{~s}$.

muscle protein synthesis while being cognizant of total energy intake, a dietary plan was proposed that includes $25-30 \mathrm{~g}$ of high-quality protein per meal. ${ }^{38)}$ As it is difficult for the elderly to eat over $20 \mathrm{~g}$ of such protein at each meal, nutrition in small quantity must be achieved. Recent evidence shows that many of the signaling components involved in sphingomyelin metabolism are present in skeletal muscle, ${ }^{39)}$ suggesting that sphingomyelin metabolism is activated in exercised muscle. In addition, sphingosine 1-phosphate and sphingosine, which are sphingomyelin metabolites, have been shown to have protective effects against muscle fatigue. ${ }^{40)}$ Although sphingomyelin has the potential to build muscle, its absorption is limited. ${ }^{20,21)}$

In this study, we prepared SM-lipo to enhance the absorption of sphingomyelin and evaluated them in both in vitro and in vivo experiments. Firstly, we prepared SM-lipo by thin lipid-film hydration. Although only sphingomyelin formed liposomes without the addition of any other lipids, these liposomes with a gel-phase bilayer due to their high phasetransition temperature may not interact strongly with the fluidphase plasma membrane of the cells in the GI tract. Therefore, we used cholesterol to make the fluid-phase of SM-lipo more stable. SM-lipo, composed of sphingomyelin-cholesterol $(7: 3$ as a molar ratio), was stable at $25^{\circ} \mathrm{C}$ at least for $30 \mathrm{~d}$ (Fig. 1). Cholesterol is often included in the liposome formulation to give further rigidity to the fluid bilayer, which contributes to both in vitro and in vivo stability of liposomes.

It is generally known that orally administered phospholipids are degraded by phospholipase, absorbed into the lymphatic vessels of the small intestines, and then transferred to the bloodstream. It is important for enhancing the oral absorption of liposomes that they be stable in the presence of gastric liquid and intestinal fluids. Particle diameter and zeta-potential of SM-lipo incubated in water, PBS, gastric liquid model or intestinal fluids model were not changed (Fig. 2). These data indicated that SM-lipo were stable in vivo after oral administration.

Subsequently, we demonstrated the absorption of sphingomyelin in the small intestine model using Caco-2 cells. Such cell culture models are of current interest for the prediction of oral drug absorption and have been used in studies on the effects of absorption enhancers. ${ }^{41)}$ Caco-2 cells have been recommended for studies on both active and passive drug transport in the presence of nutrients and other chemicals. ${ }^{42)}$ The effects of substances such as absorption enhancers on this layer can be easily studied. ${ }^{42,43)}$ The penetration by sphingomyelin formulated in liposomes was $15.4 \%$, whereas there was little penetration by free sphingomyelin (Fig. 3). Also, we checked the penetration of sphingomyelin by using PAMPA. This technique involves no cell culture. PAMPA uses 2 aqueous buffer solution wells separated by an artificial membrane. The artificial membrane consists of a lipid in organic diluent. ${ }^{44)}$ Thus, PAMPA can easily evaluate the absorption of drugs or nutrition. Since PAMPA features a single permeability mechanism (passive diffusion), whereas Caco-2 cells have a greater variety of permeability mechanisms, PAMPA is effective for measuring the absorption when passive diffusion is predominant. ${ }^{45)}$ In the PAMPA study, the percent membrane absorption of sphingomyelin with SM-lipo was higher than that with dispersed sphingomyelin. These results indicated that the liposome formulation was effective for absorption across both Caco-2 cell monolayers and PAMPA.

As affirmation of the effect of the SM-lipo formulation on sphingomyelin penetration, we evaluated the effect of these liposomes on the proliferation of muscle cell in vitro. We selected the murine $\mathrm{C} 2 \mathrm{C} 12$ cells, which grow as proliferating myoblasts. Recent microarray studies on $\mathrm{C} 2 \mathrm{C} 12$ cells have afforded a broad molecular description of myogenesis and identified sets of genes that display transcriptional variations in expression between proliferating and differentiating cells. ${ }^{46-48)}$ Another report showed that the treatment of stretched $\mathrm{C} 2 \mathrm{C} 12$ cells with sphingomyelin significantly increased Dok-7 gene expression. ${ }^{49)}$ When we evaluated the proliferation rate of C2C12 cells treated with SM-lipo, the results revealed that these murine $\mathrm{C} 2 \mathrm{C} 12$ myoblasts showed greater cell proliferation than those treated with sphingomyelin or PC-lipo (Fig. 4). Androgens are modulators of skeletal muscle adaptation and regeneration processes, and the differentiation and proliferation of $\mathrm{C} 2 \mathrm{C} 12$ cells is known to be stimulated by androgens and associated with the modulation of myostatin and Pax7 expression. ${ }^{50)}$ So this result can be understood to indicate that 
A

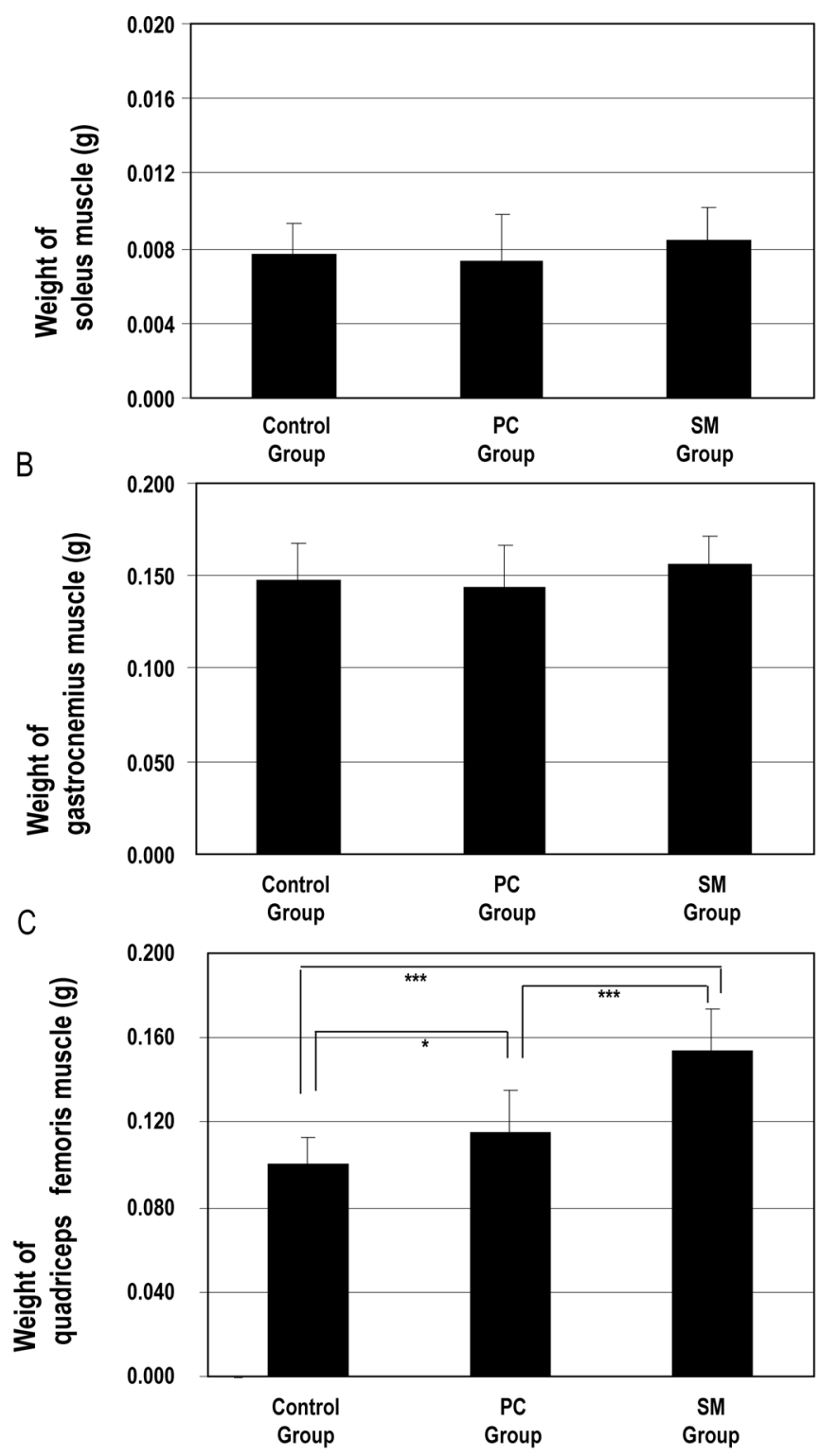

Fig. 6. Effect of Sphingomyelin-Based Liposomes on Right-Leg Muscle Mass after 10 Weeks of Intake

SAMP1 were cared for as described in the legend of Fig. 5: Each group was given access to plain drinking water $(\boldsymbol{\square})$ or to drinking water containing $2 \mathrm{~mm} \mathrm{SM-}$ lipo $(\mathbf{)})$ or $2 \mathrm{~mm}$ PC-lipo $(\mathbf{\Delta})$ for 10 weeks. Then, the mice were sacrificed and the indicated muscles were removed. The weights of soleus muscle (A), gastrocnemius muscle (B), and quadriceps femoris muscle (C) are shown. Results are shown as the mean \pm S.D. Significant difference is shown as follows: $* p<0.05, * * * p<0.001$.

SM-lipo were highly taken up into $\mathrm{C} 2 \mathrm{C} 12$ cells and enhanced the proliferation of these cells.

Finally, we demonstrated the effect of SM-lipo on muscle in vivo by using SAMP1. As a result, SAMP1 that had ingested SM-lipo tended to demonstrate an extended swimming time until fatigue compared with SAMP1 with PC-lipo or water intake (Fig. 5). Additionally, SAMP1 having ingested SMlipo also showed a significant increase in the mass of their quadriceps femoris muscle compared with SAMP1 that had ingested PC-lipo or water (Fig. 6C). In contrast, there was no significant difference in the weight of the soleus or gastrocnemius muscle between the SM-lipo group and other groups (Figs. 6A, B). These results indicated that long-term feeding with SM-lipo was effective in increasing the mass of the quadriceps femoris of SAMP1 mice. The differences in mass gain between the muscles after the SM-lipo treatment appear to be related to the differences in myosin heavy chain (MHC) isoforms expressed in each muscle. ${ }^{51)}$ Sphingosine-1-phosphate might stimulate the secretion of insulin-like growth factor-1 (IGF-1), a growth factor which activates the transcription of MHC IIB and X in satellite cells. ${ }^{52,53)}$ Because the expression of MHC IIB and X was shown to be higher in quadriceps femoris muscle than in soleus or gastrocnemius muscle, ${ }^{51)}$ it is possible that the SM-lipo treatment resulted in significant mass gain of quadriceps femoris muscle via IGF-1 signaling activation. Although the reason for increase in the weight of quadriceps femoris muscle in the PC-lipo group (Fig. 6C) is unclear, phosphatidylcholine might support cell membrane expansion during muscle growth. ${ }^{54)}$

In conclusion, SM-lipo increased the transport of sphingomyelin into Caco-2 cells and up-regulated the proliferation of murine $\mathrm{C} 2 \mathrm{C} 12$ myoblasts. Moreover, SAMP1 mice that had ingested SM-lipo demonstrated a significantly increased mass of their quadriceps femoris muscle and an extended swimming time. These results suggest that SM-lipo enhanced the absorption of sphingomyelin and increased muscle mass both in vitro and in vivo, thus making them a candidate for protection against or treatment of sarcopenia.

Conflict of Interest The authors declare no conflict of interest.

Supplementary Materials The online version of this article contains supplementary materials.

Supplementary Fig. 1. Absorption of Sphingomyelin or Sphingomyelin-Based Liposomes Assessed by the Parallel Artificial Membrane Permeability Assay (PAMPA)

Sphingomyelin (ם) or SM-lipo (@) were added to each well of a 96-well plate and an insert having an artificial membrane was set on each well with filling water. After incubation for $24 \mathrm{~h}$, the residual amount of SM in the lower compartment was determined with HPLC. The experiment was repeated 3 times, and results shown are the mean values.

Supplementary Fig. 2. Body-Weight Change and Water Intake during the Experiment

Each group was given access to plain drinking water (ם) or drinking water containing $2 \mathrm{~mm}$ SM-lipo (-) or $2 \mathrm{~mm}$ PClipo (\) for 10 weeks. The body weight of each mouse was monitored weekly (a). The amount of loss of plain water or water containing liposomes in each cage (three mice) was determined once a week (b). The results shown are the mean value \pm S.D.

\section{REFERENCES}

1) Janssen I, Shepard DS, Katzmarzyk PT, Roubenoff R. The health care costs of sacopenia in the United States. J. Am. Geriatr. Soc., 52, 80-85 (2004).

2) Sinha-Hikim I, Sinha-Hikim AP, Parveen M, Shen R, Goswami R, Tran P, Crum A, Norris KC. Long-term supplementation with a cystine-based antioxidant delays loss of muscle mass in aging. $J$. Gerontol. A Biol. Sci. Med. Sci., 68, 749-759 (2013).

3) Tan LJ, Liu SL, Lei SF, Papasian CJ, Deng HW. Molecular genetic studies of gene identification for sarcopenia. Hum. Genet., 131, 1-31 
(2012).

4) Vincent G, Lamon S, Gant N, Vincent PJ, MacDonald JR, Markworth JF, Edge JA, Hickey AJ. Changes in mitochondrial function and mitochondria associated protein expression in response to 2-weeks of high intensity interval training. Front. Physiol., 6, 51 (2015).

5) Casperson SL, Sheffield-Moore M, Hewlings SJ, Paddon-Jones D. Leucine supplementation chronically improves muscle protein synthesis in older adults consuming the RDA for protein. Clin. Nutr., 31, 512-519 (2012).

6) Deldicque L, Louis M, Theisen D, Nielens H., Denhoux M, Thissen J-P, Rennie MJ, Francaux M. Increased IGF mRNA in human skeletal muscle after creatine supplementation. Med. Sci. Sports Exerc., 37, 731-736 (2005)

7) Katsanos CS, Chinkes DL, Paddon-Jones D, Zhang XJ, Aarsland A, Wolfe RR. Whey protein ingestion in elderly persons results in greater muscle protein accrual than ingestion of its constituent essential amino acid content. Nutr. Res., 28, 651-658 (2008).

8) Thudichum JLW. A treatise on the chemical constitution of brain. Bailliere, Tindall, and Cox, London, U.K. (1884).

9) Vesper H, Schmelz EM, Nikolova-Karakashian MN, Dillehay DL, Lynch DV, Merrill AH Jr. Sphingolipids in food and the emerging importance of sphingolipids to nutrition. J. Nutr., 129, 1239-1250 (1999).

10) Zeisel SH, Char D, Sheard NF. Choline, phosphatidylcholine and sphingomyelin in human and bovine milk and infant formulas. $J$. Nutr., 116, 50-58 (1986).

11) Zeisel SH, Mar MH, Howe JC, Holden JM. Concentrations of choline-containing compounds and betaine in common foods. J. Nutr., 133, 1302-1307 (2003)

12) Morita SY, Kawabe M, Sakurai A, Okuhira K, Vertut-Doï A, Nakano M, Handa T. Ceramide in lipid particles enhances heparin sulfate proteoglycan and low density lipoprotein receptor-related protein-mediated uptake by macrophages. J. Biol. Chem., 279, 24355-24361 (2004).

13) Holland WL, Summers SA. Sphingolipids, insulin resistance, and metabolic disease: new insights from in vivo manipulation of sphingolipid metabolism. Endocr. Rev., 29, 381-402 (2008).

14) Hla T, Dannenberg AJ. Sphingolipid signaling in metabolic disorders. Cell Metab., 16, 420-434 (2012).

15) Haramizu S, Ota N, Otsuka A, Hashizume K, Sugita S, Hase T, Murase T, Shimotoyodome A. Dietary milk fat globule membrane improves endurance capacity in mice. Am. J. Physiol. Regul. Integr. Comp. Physiol., 307, R1009-R1017 (2014).

16) Sabbadini RA, Danieli-Betto D, Betto R. The role of sphingolipids in the control of skeletal muscle function: a review. Ital. J. Neurol. Sci., 20, 423-430 (1999).

17) Nagata Y, Partridge TA, Matsuda R, Zammit PS. Entry of muscle satellite cells into the cell cycle requires sphingolipid signaling. $J$. Cell Biol., 174, 245-253 (2006).

18) Rosenfeldt HM, Amrani Y, Watterson KR, Murthy KS, Panettieri RA Jr, Spiegel S. Sphingosine-1-phosphate stimulates contraction of human airway smooth muscle cells. FASEB J., 17, 1789-1799 (2003).

19) Danieli-Betto D, Germinario E, Esposito A, Megighian A, Midrio M, Ravara B, Damiani E, Libera LD, Sabbadini RA, Betto R. Sphingosine 1-phosphate protects mouse extensor digitorum longus skeletal muscle during fatigue. Am. J. Physiol. Cell Physiol., 288, C1367-C1373 (2005)

20) Nilsson A, Duan RD. Absorption and lipoprotein transport of sphingomyelin. J. Lipid Res., 47, 154-171 (2006).

21) Nyberg L, Nilsson A, Lundgren P, Duan RD. Localization and capacity of sphingomyelin digestion in the rat intestinal tract. $J$. Nutr. Biochem., 8, 112-118 (1997).

22) Nilsson A. Metabolism of sphingomyelin in the intestinal tract of the rat. Biochim. Biophys. Acta, 164, 575-584 (1968).
23) Iwanaga $\mathrm{K}$, Ono S, Narioka K, Kakemi M, Morimoto K, Yamashita S, Namba Y, Oku N. Application of surface-coated liposomes for oral delivery of peptide: effects of coating the liposome's surface on the GI transit of insulin. J. Pharm. Sci., 88, 248-252 (1999).

24) Thongborisute J, Takeuchi H, Yamamoto H, Kawashima Y. Visualization of the penetrative and mucoadhesive properties of chitosan and chitosan-coated liposomes through the rat intestine. J. Liposome Res., 16, 127-141 (2006).

25) Sugihara H, Yamamoto H, Kawashima Y, Takeuchi H. Effectiveness of submicronized chitosan-coated liposomes in oral absorption of indomethacin. J. Liposome Res., 22, 72-79 (2012).

26) Malmsten M, Bergenståhl B, Nyberg L, Odham G. Sphingomyelin from milk-characterization of liquid crystalline, liposome and emulsion properties. J. Am. Oil Chem. Soc., 71, 1021-1026 (1994).

27) Tokudome Y, Uchida R, Yokote T, Todo H, Hada N, Kon T, Yasuda J, Hayashi H, Hashimoto F, Sugibayashi K. Effect of topically applied sphingomyelin-based liposomes on the ceramide level in a three-dimensional cultured human skin model. J. Liposome Res., 20, 49-54 (2010).

28) Kisel MA, Kulik LN, Tsybovsky IS, Vlasov AP, Vorob'yov MS, Kholodova EA, Zabarovskaya ZV. Liposomes with phosphatidylethanol as a carrier for oral delivery of insulin: studies in the rat. Int. J. Pharm., 216, 105-114 (2001).

29) Pinto M. Enterocyte-like differentiation and polarization of the human colon carcinoma cell line Caco-2 in culture. Biol. Cell, 47, 323-330 (1983).

30) Hidalgo IJ, Raub TJ, Borchardt RT. Characterization of the human colon carcinoma cell line (Caco-2) as a model system for intestinal epithelial permeability. Gastroenterology, 96, 736-749 (1989).

31) Rodríguez-Alcalá LM, Fontecha J. Major lipid classes separation of buttermilk, and cows, goats and ewes milk by high performance liquid chromatography with an evaporative light scattering detector focused on the phospholipid fraction. J. Chromatogr. A, 1217, 3063-3066 (2010).

32) Baumgartner RN, Koehler KM, Gallagher D, Romero L, Heymsfield SB, Ross RR, Garry PJ, Lindeman RD. Epidemiology of sarcopenia among the elderly in New Mexico. Am. J. Epidemiol., 147, 755-763 (1998).

33) Cruz-Jentoft AJ, Baeyens JP, Bauer JM, Boirie Y, Cederholm T, Landi F, Martin FC, Michel JP, Rolland Y, Schneider SM, Topinková E, Vandewoude M, Zamboni M, European Working Group on Sarcopenia in Older People. Sarcopenia: European consensus on definition and diagnosis: Report of the European Working Group on Sarcopenia in Older People. Age Ageing, 39, 412-423 (2010).

34) Walrand S, Guillet C, Salles J, Cano N, Boirie Y. Physiopathological mechanism of sarcopenia. Clin. Geriatr. Med., 27, 365-385 (2011).

35) Koopman R, van Loon LJ. Aging, exercise and muscle protein metabolism. J. Appl. Physiol., 106, 2040-2048 (2009).

36) Rolland Y, Czerwinski S, Abellan Van Kan G, Morley JE, Cesari M, Onder G, Woo J, Baumgartner R, Pillard F, Boirie Y, Chumlea WM, Vellas B. Sacropenia: it's assessment, etiology, pathogenesis, consequences and future perspectives. J. Nutr. Health Aging, 12, 433-450 (2008)

37) Yarasheski KE. Exercise, aging and muscle protein metabolism. $J$. Gerontol. A Biol. Sci. Med. Sci., 58, M918-M922 (2003).

38) Paddon-Jones D, Rasmussen BB. Dietary protein recommendations and the prevention of sarcopenia. Curr. Opin. Clin. Nutr. Metab. Care, 12, 86-90 (2009).

39) Sabbadini RA, Danieli-Betto D, Betto R. The role of sphingolipids in the control of skeletal muscle function. Ital. J. Neurol. Sci., 20, 423-430 (1999)

40) Danieli-Betto D, Germinario E, Esposito A, Megighian A, Midrio M, Ravara B, Damiani E, Libera LD, Sabbadini RA, Betto R. Sphingosine 1-phosphate protects mouse extensor digitorum longus 
skeletal muscle during fatigue. Am. J. Physiol. Cell Physiol., 288, C1367-C1373 (2005)

41) Artursson P. Cell cultures as models for drug absorption across the intestinal mucosa. Crit. Rev. Ther. Drug Carrier Syst., 8, 305-330 (1991).

42) Hovgaard L, Brondsted H, Nielsen HM. Drug delivery studies in caco-2 monolayer. II. absorption enhancer effects of lysophosphatidylcholines. Int. J. Pharm., 114, 141-149 (1995).

43) Johansson F, Hjertberg E, Eirefelt S, Tronde A, Hultkvist Bengtsson U. Mechanism for absorption enhancement of inhaled insulin by sodium taurocholate. Eur. J. Pharm. Sci., 17, 63-71 (2002).

44) Kansy M, Senner F, Gubernator K. Physicochemical high throughput screening: Parallel artificial membrane permeation assay in the description of passive absorption processes. J. Med. Chem., 41, 1007-1010 (1998).

45) Kerns EH, Di L, Petusky S, Farris M, Ley R, Jupp P. Combined application of parallel artificial membrane permeability assay and Caco-2 permeability assays in drug discovery. J. Pharm. Sci., 93, 1440-1453 (2004).

46) Moran JL, Li Y, Hill AA, Mounts WM, Miller CP. Gene expression changes during mouse skeletal myoblast differentiation revealed by transcriptional profiling. Physiol. Genomics, 10, 103-111 (2002).

47) Delgado I, Huang X, Jones S, Zhang L, Hatcher R, Gao B, Zhang P. Dynamic gene expression during the onset of myoblast differentiation in vitro. Genomics, 82, 109-121 (2003).

48) Tomczak KK, Marinescu VD, Ramoni MF, Sanoudou D, Montanaro
F, Han M, Kunkel LM, Kohane IS, Beggs AH. Expression profiling and identification of novel genes involved in myogenic differentiation. FASEB J., 18, 403-405 (2004).

49) Haramizu S, Mori T, Yano M, Ota N, Hashizume K, Otsuka A, Hase T, Shimotoyodome A. Habitual exercise plus dietary supplementation with milk fat globule membrane improves muscle function deficits via neuromuscular development in senescence-accelerated mice. SpringerPlus, 3, 339 (2014)

50) Diel P, Baadners D, Schlüpmann K, Velders M, Schwarz JP. C2C12 myoblastoma cell differentiation and proliferation is stimulated by androgens and associated with a modulation of myostatin and Pax7 expression. J. Mol. Endocrinol., 40, 231-241 (2008).

51) Carlson CJ, Booth FW, Gordon SE. Skeletal muscle myostatin mRNA expression is fiber-type specific and increases during hindlimb unloading. Am. J. Physiol., 277, R601-R606 (1999).

52) Verdijk LB, Gleeson BG, Jonkers RA, Meijer K, Savelberg HH, Dendale P, van Loon LJ. Skeletal muscle hypertrophy following resistance training is accompanied by a fiber type-specific increase in satellite cell content in elderly men. J. Gerontol. A Biol. Sci. Med. Sci., 64A, 332-339 (2009).

53) Bernacchioni C, Cencetti F, Blescia S, Donati C, Bruni P. Sphingosine kinase/sphingosine 1-phosphate axis: a new player for insulin-like growth factor-1-induced myoblast differentiation. Skelet. Muscle, 2, 15 (2012).

54) Jäger R, Purpura M, Kingsley M. Phospholipids and sports performance. J. Int. Soc. Sports Nutr., 4, 5 (2007). 